\title{
Inequalities for fractional Riemann-Liouville integrals of certain class of convex functions
}

\author{
Ghulam Farid ${ }^{1}$, Josip Pečarić ${ }^{2}$ and Kamsing Nonlaopon ${ }^{3 *}$ (I)
}

\author{
"Correspondence: nkamsi@kku.ac.th \\ ${ }^{3}$ Department of Mathematics, \\ Faculty of Science, Khon Kaen \\ University, Khon Kaen 40002, \\ Thailand \\ Full list of author information is \\ available at the end of the article
}

\begin{abstract}
Fractional calculus operators play a very important role in generalizing concepts of calculus used in diverse fields of science. In this paper, we use Riemann-Liouville fractional integrals to establish generalized identities, which are further applied to obtain midpoint and trapezoidal inequalities for convex function with respect to a strictly monotone function. These inequalities reproduce midpoint and trapezoidal inequalities for convex, harmonic convex, $p$-convex, and geometrically convex functions. Also, some new inequalities can be generated via specific strictly monotone functions.
\end{abstract}

MSC: $26 \mathrm{~A} 51 ; 26 \mathrm{D} 15$

Keywords: Convex function; Hadamard inequality; Riemann-Liouville fractional integrals; Error bounds

\section{Introduction}

A convex function $\psi:[a, b] \rightarrow \mathbb{R}$ satisfies the inequality

$$
\psi(t x+(1-t) y) \leq t \psi(x)+(1-t) \psi(y),
$$

for $t \in[0,1]$, and $x, y \in[a, b]$.

If an additive inverse of $\psi$ is convex, then $\psi$ would be a concave function. Recently, several integral inequalities relating to many well-known classes of convex functions have been proven using different concepts and techniques. Generalizations of convex functions have played an important role in the development of several fields of pure and applied sciences. Convexity theory has been further extended in different directions with innovative techniques. The Hermite-Hadamard inequality given below is a tangible geometric visualization of a convex function.

Theorem 1 ([7]) Let $\psi:[a, b] \rightarrow \mathbb{R}$ be a convex function. Then we have the following inequality:

$$
\psi\left(\frac{a+b}{2}\right) \leq \frac{1}{b-a} \int_{a}^{b} \psi(x) d x \leq \frac{\psi(a)+\psi(b)}{2} .
$$

If function $\psi$ is concave, then inequality (1.1) will hold in the reverse direction.

(c) The Author(s) 2022. This article is licensed under a Creative Commons Attribution 4.0 International License, which permits use sharing, adaptation, distribution and reproduction in any medium or format, as long as you give appropriate credit to the original author(s) and the source, provide a link to the Creative Commons licence, and indicate if changes were made. The images or other third party material in this article are included in the article's Creative Commons licence, unless indicated otherwise in a credit line to the material. If material is not included in the article's Creative Commons licence and your intended use is not permitted by statutory regulation or exceeds the permitted use, you will need to obtain permission directly from the copyright holder. To view a copy of this licence, visit http://creativecommons.org/licenses/by/4.0/. 
Error estimations of the Hermite-Hadamard inequality give the error bounds of the midpoint and trapezoidal quadrature rules. The inequality stated in (1.1) has been studied for different kinds of convex functions, also by establishing some identities their error estimates are obtained. For a detailed study we refer the readers to $[1-5,8,9,12,13,15,16]$. The aim of this paper is to present error estimates of Hermite-Hadamard-type inequalities for Riemann-Liouville fractional integrals of different kinds of convex functions in generalized form by applying convexity of a function with respect to a strictly monotone function.

Definition 1 ([18]) If $\phi$ is a strictly monotone function, then a function $\psi$ is said to be convex with respect to $\phi$ if $\psi \circ \phi^{-1}$ is a convex function.

The above definition generates convex, harmonic convex, $p$-convex, and geometrically convex functions corresponding to the identity, reciprocal, power, and logarithm functions, respectively, see [25]. Next, we define Riemann-Liouville fractional integrals.

Definition 2 ([11]) Let $f \in L_{1}[a, b]$. Then left- and right-sided Riemann-Liouville fractional integrals of a function $f$ of order $\mu$, where $\Re(\mu)>0$, are defined as follows:

$$
I_{a^{+}}^{\mu} f(x)=\frac{1}{\Gamma(\mu)} \int_{a}^{x}(x-t)^{\mu-1} f(t) d t, \quad x>a,
$$

and

$$
I_{b}^{\mu} f(x)=\frac{1}{\Gamma(\mu)} \int_{x}^{b}(t-x)^{\mu-1} f(t) d t, \quad x<b
$$

Many inequalities have been analyzed for different types of convex functions using Riemann-Liouville fractional integrals [14, 20, 21, 23]. Our aim in this paper is to utilize Riemann-Liouville fractional integrals to investigate the Hermite-Hadamard inequality for a convex function with respect to a strictly monotone function. The error estimation of this inequality provides midpoint- and trapezoidal-type inequalities for convex, geometrically convex, harmonically convex, and $p$-convex functions. Next, we give the HermiteHadamard inequality for a convex function with respect to strictly monotone function.

Theorem 2 ([24]) Let I, J be intervals in $\mathbb{R}$ and $\psi:[a, b] \subset I \rightarrow \mathbb{R}$ a convex function, also let $\phi: J \supset[a, b] \rightarrow \mathbb{R}$ be a strictly monotone function. If $\psi$ is convex with respect to $\phi$, then the following inequality holds:

$$
\psi\left(\phi^{-1}\left(\frac{\phi(a)+\phi(b)}{2}\right)\right) \leq \frac{1}{\phi(b)-\phi(a)} \int_{\phi(a)}^{\phi(b)} \psi\left(\phi^{-1}(t)\right) d t \leq \frac{\psi(a)+\psi(b)}{2} .
$$

In the upcoming section, we establish a trapezoidal type identity for a function via another strictly monotone function. By applying the established identity, the trapezoidaltype inequalities are studied using convexity. Moreover, a midpoint-type identity for a function via another strictly monotone function is established, and corresponding inequalities are studied. The findings of this article are connected with the inequalities proved in $[6,8,10,12,17,20-22]$. 


\section{Error estimation of Hermite-Hadamard inequality for a convex function with respect to a strictly monotone function}

First, we establish the following integral identity to study the error estimates of the Hermite-Hadamard inequality (1.4).

Lemma 1 Let $a, b \in \mathbb{R}$ with $a<b$ and $\psi:[a, b] \rightarrow \mathbb{R}$ be a function, also let $\phi:[a, b] \rightarrow \mathbb{R}$ be a strictly monotone function such that $\psi \circ \phi^{-1}$ is differentiable and $\left(\psi \circ \phi^{-1}\right)^{\prime} \in L[a, b]$. Then the following identity holds:

$$
\begin{aligned}
& \frac{\psi(a)+\psi(b)}{2}-\frac{\Gamma(\mu+1)}{2(\phi(b)-\phi(a))^{\mu}}\left(J_{\phi(a)^{+}}^{\mu} \psi(b)+J_{\phi(b)^{-}}^{\mu} \psi(a)\right) \\
& =\frac{\phi(b)-\phi(a)}{2} \int_{0}^{1}\left((1-t)^{\mu}-t^{\mu}\right)\left(\psi \circ \phi^{-1}\right)^{\prime}(t \phi(a)+(1-t) \phi(b)) d t .
\end{aligned}
$$

Proof First, we evaluate the following integral:

$$
\begin{aligned}
\int_{0}^{1}(1-t)^{\mu}\left(\psi \circ \phi^{-1}\right)^{\prime}(t \phi(a)+(1-t) \phi(b)) d t \\
=\left.\frac{(1-t)^{\mu}\left(\psi \circ \phi^{-1}\right)(t \phi(a)+(1-t) \phi(b))}{\phi(a)-\phi(b)}\right|_{0} ^{1} \\
\quad+\mu \int_{0}^{1} \frac{(1-t)^{\mu-1}\left(\psi \circ \phi^{-1}\right)(t \phi(a)+(1-t) \phi(b)) d t}{\phi(a)-\phi(b)} \\
=\frac{\psi(b)}{\phi(b)-\phi(a)}-\frac{\mu}{\phi(b)-\phi(a)} \int_{0}^{1}(1-t)^{\mu-1}\left(\psi \circ \phi^{-1}\right)(t \phi(a)+(1-t) \phi(b)) d t \\
=\frac{\psi(b)}{\phi(b)-\phi(a)}-\frac{\mu}{(\phi(b)-\phi(a))^{\mu+1}} \int_{\phi(a)}^{\phi(b)}(u-\phi(a))^{\mu-1}\left(\psi \circ \phi^{-1}\right)(u) d u \\
=\frac{\psi(b)}{\phi(b)-\phi(a)}-\frac{\Gamma(\mu+1)}{(\phi(b)-\phi(a))^{\mu+1}} J_{\phi(b)^{-}}^{\mu} \psi(a) .
\end{aligned}
$$

Similarly, from integration by parts one can have the following equation:

$$
\begin{aligned}
& \int_{0}^{1} t^{\mu}\left(\psi \circ \phi^{-1}\right)^{\prime}(t \phi(a)+(1-t) \phi(b)) d t \\
& \quad=\frac{-\psi(a)}{\phi(b)-\phi(a)}+\frac{\Gamma(\mu+1)}{(\phi(b)-\phi(a))^{\mu+1}} J_{\phi(a)^{+}}^{\mu} \psi(b) .
\end{aligned}
$$

By using (2.2) and (2.3) in the right-hand side of (2.1), the left-hand side can be obtained.

Remark 1 By setting $\phi(x)=x$, identity (2.1) reduces to Lemma 2 of [20]; for $\phi(x)=x, \mu=$ 1, identity (2.1) provides Lemma 2.1 of [6]. By setting $\phi(x)=\frac{1}{x}$, identity (2.1) reduces to Lemma 3 of [10]; for $\phi(x)=\frac{1}{x}, \mu=1$, identity (2.1) provides Lemma 2.5 of [8]. By setting $\phi(x)=x^{r}$, identity (2.1) reduces to Lemma 2.1 for Riemann-Liouville fractional integrals of [22]; for $\phi(x)=x^{r}, \mu=1$, identity (2.1) provides Lemma 2.4 of [17]. By considering other strictly monotone functions in place of $\phi$, many other corresponding identities can be formulated. 
By using Lemma 1, we prove the following error estimate of Theorem 2.

Theorem 3 Let $a, b \in \mathbb{R}$ with $a<b$ and $\psi:[a, b] \rightarrow \mathbb{R}$ be a function, also let $\phi:[a, b] \rightarrow \mathbb{R}$ be a strictly monotone function such that $\psi \circ \phi^{-1}$ is differentiable and $\left(\psi \circ \phi^{-1}\right)^{\prime} \in L[a, b]$. If $\left|\left(\psi \circ \phi^{-1}\right)^{\prime}\right|$ is convex, then the following inequality holds:

$$
\begin{aligned}
& \left|\frac{\psi(a)+\psi(b)}{2}-\frac{\Gamma(\mu+1)}{2(\phi(b)-\phi(a))^{\mu}}\left(J_{\phi(a)^{+}}^{\mu} \psi(b)+J_{\phi(b)^{-}}^{\mu} \psi(a)\right)\right| \\
& \leq \frac{|\phi(b)-\phi(a)|}{2(\mu+1)}\left(1-\frac{1}{2^{\mu}}\right)\left\{\left|\left(\psi \circ \phi^{-1}\right)^{\prime}(\phi(a))\right|+\left|\left(\psi \circ \phi^{-1}\right)^{\prime}(\phi(b))\right|\right\} .
\end{aligned}
$$

Proof By using the property of the absolute value function in Lemma 1, one can get the following inequality:

$$
\begin{aligned}
& \left|\frac{\psi(a)+\psi(b)}{2}-\frac{\Gamma(\mu+1)}{2(\phi(b)-\phi(a))^{\mu}}\left(J_{\phi(a)^{+}}^{\mu} \psi(b)+J_{\phi(b)^{-}}^{\mu} \psi(a)\right)\right| \\
& \quad \leq \frac{|\phi(b)-\phi(a)|}{2} \int_{0}^{1}\left|(1-t)^{\mu}-t^{\mu}\right|\left|\left(\psi \circ \phi^{-1}\right)^{\prime}(t \phi(a)+(1-t) \phi(b))\right| d t .
\end{aligned}
$$

Now, by using convexity of $\left|\left(\psi \circ \phi^{-1}\right)^{\prime}\right|$ on the right-hand side of the above inequality (2.5), we get the following inequality:

$$
\begin{aligned}
\mid \frac{\psi(a)}{2}+\psi(b) & \frac{\Gamma(\mu+1)}{2(\phi(b)-\phi(a))^{\mu}}\left(J_{\phi(a)^{+}}^{\mu} \psi(b)+J_{\phi(b)^{-}}^{\mu} \psi(a)\right) \mid \\
\leq & \frac{|\phi(b)-\phi(a)|}{2} \\
& \times \int_{0}^{1}\left|(1-t)^{\mu}-t^{\mu}\right|\left(t\left|\left(\psi \circ \phi^{-1}\right)^{\prime}(\phi(a))\right|+(1-t)\left|\left(\psi \circ \phi^{-1}\right)^{\prime}(\phi(b))\right|\right) d t \\
\leq & \frac{|\phi(b)-\phi(a)|}{2} \\
& \times \int_{0}^{\frac{1}{2}}\left((1-t)^{\mu}-t^{\mu}\right)\left(t\left|\left(\psi \circ \phi^{-1}\right)^{\prime}(\phi(a))\right|+(1-t)\left|\left(\psi \circ \phi^{-1}\right)^{\prime}(\phi(b))\right|\right) d t \\
& +\int_{\frac{1}{2}}^{1}\left(t^{\mu}-(1-t)^{\mu}\right)\left(t\left|\left(\psi \circ \phi^{-1}\right)^{\prime}(\phi(a))\right|+(1-t)\left|\left(\psi \circ \phi^{-1}\right)^{\prime}(\phi(b))\right|\right) d t \\
= & \frac{|\phi(b)-\phi(a)|}{2}\left(\left|\left(\psi \circ \phi^{-1}\right)^{\prime}(\phi(a))\right| \int_{0}^{\frac{1}{2}}\left(t(1-t)^{\mu}-t^{\mu+1}\right) d t+\left|\left(\psi \circ \phi^{-1}\right)^{\prime}(\phi(b))\right|\right. \\
& \times \int_{0}^{\frac{1}{2}}\left((1-t)^{\mu+1}-t^{\mu}(1-t) d t+\int_{\frac{1}{2}}^{1}\left(t^{\mu+1}-t(1-t)^{\mu}\right) d t+\left|\left(\psi \circ \phi^{-1}\right)^{\prime}(\phi(b))\right|\right. \\
& \times \int_{\frac{1}{2}}^{1}\left(t^{\mu}(1-t)-(1-t)^{\mu+1} d t\right),
\end{aligned}
$$

from which, after a little computation, one can get (2.4). 
Corollary 1 By setting $\phi(x)=\frac{1}{x}$, inequality (2.4) reduces to the following inequality:

$$
\begin{aligned}
& \left|\frac{\psi(a)+\psi(b)}{2}-\frac{\Gamma(\mu+1)}{2}\left(\frac{a b}{b-a}\right)^{\mu}\left(J_{\left(\frac{1}{a}\right)^{-}}^{\mu} \psi \circ g\left(\frac{1}{b}\right)+J_{\left(\frac{1}{b}\right)^{+}}^{\mu} \psi \circ g\left(\frac{1}{a}\right)\right)\right| \\
& \leq \frac{b-a}{2 a b(\mu+1)}\left\{a^{2}\left|\psi^{\prime}(a)\right|+b^{2}\left|\psi^{\prime}(b)\right|\right\},
\end{aligned}
$$

where $g(t)=\frac{1}{t}$.

Corollary 2 By setting $\phi(x)=\frac{1}{x}$ and $\mu=1$, inequality (2.4) reduces to the following inequality:

$$
\left|\frac{\psi(a)+\psi(b)}{2}-\frac{a b}{(b-a)} \int_{\frac{1}{b}}^{\frac{1}{a}}(\psi \circ g)(t) d t\right| \leq \frac{b-a}{8 a b}\left\{a^{2}\left|\psi^{\prime}(a)\right|+b^{2}\left|\psi^{\prime}(b)\right|\right\},
$$

where $g(t)=\frac{1}{t}$.

Corollary 3 By setting $\phi(x)=x^{r}, r \neq 0$, inequality (2.4) reduces to the following inequality:

$$
\begin{aligned}
& \left|\frac{\psi(a)+\psi(b)}{2}-\frac{r^{\mu} \Gamma(\mu+1)}{b^{r}-a^{r}}\left({ }^{r} J_{a^{+}}^{\mu} \psi(z)+{ }^{r} J_{b^{-}}^{\mu} \psi(z)\right)\right| \\
& \leq \frac{\left|b^{r}-a^{r}\right|}{2|r|(\mu+1)}\left(1-\frac{1}{2^{\mu}}\right)\left\{a^{1-p}\left|\psi^{\prime}(a)\right|+\psi^{1-p}\left|f^{\prime}(b)\right|\right\} .
\end{aligned}
$$

Corollary 4 By setting $\phi(x)=x^{r}, r \neq 0$ and $\mu=1$, inequality (2.4) reduces to the following inequality:

$$
\left|\frac{\psi(a)+\psi(b)}{2}-\frac{r^{2}}{b^{r}-a^{r}} \int_{a}^{b} t^{r-1} f(t) d t\right| \leq \frac{\left|b^{r}-a^{r}\right|}{8|p|}\left\{a^{1-r}\left|\psi^{\prime}(a)\right|+\psi^{1-r}\left|\psi^{\prime}(b)\right|\right\} .
$$

Corollary 5 By setting $\phi(x)=\ln x$, inequality (2.4) reduces to the following inequality:

$$
\begin{aligned}
& \left|\frac{\psi(a)+\psi(b)}{2}-\frac{\Gamma(\mu+1)}{2(\ln b-\ln a)^{\mu}}\left(J_{(\ln a)^{+}}^{\mu} \psi(b)+J_{(\ln b)^{-}}^{\mu} \psi(a)\right)\right| \\
& \leq \frac{\ln b-\ln a}{2(\mu+1)}\left(1-\frac{1}{2^{\mu}}\right)\left\{a\left|\psi^{\prime}(a)\right|+b\left|\psi^{\prime}(b)\right|\right\} .
\end{aligned}
$$

Corollary 6 By setting $\phi(x)=\ln x$ and $\mu=1$, inequality (2.4) reduces to the following inequality:

$$
\left|\frac{\psi(a)+\psi(b)}{2}-\frac{1}{(\ln b-\ln a)} \int_{a}^{b} \frac{\psi(u)}{u} d u\right| \leq \frac{\ln b-\ln a}{8}\left\{a\left|\psi^{\prime}(a)\right|+b\left|\psi^{\prime}(b)\right|\right\} .
$$

Remark 2 By setting $\phi(x)=x$, inequality (2.4) reduces to the inequality proved in Theorem 3 of [20]. By setting $\phi(x)=x$ and $\mu=1$, inequality (2.4) reduces to the inequality proved in Theorem 2.2 of [6].

Remark 3 By considering other strictly monotone functions in place of $\phi$ in Theorem 3, the corresponding inequalities can be formulated. 
Theorem 4 Let $a, b \in \mathbb{R}$ with $a<b$ and $\psi:[a, b] \rightarrow \mathbb{R}$ be a function, also let $\phi:[a, b] \rightarrow \mathbb{R}$ be a strictly monotone function such that $\psi \circ \phi^{-1}$ is differentiable and $\left(\psi \circ \phi^{-1}\right)^{\prime} \in L[a, b]$. If $\left|\left(\psi \circ \phi^{-1}\right)^{\prime}\right|^{q}, q \geq 1$ is convex, then the following inequality holds:

$$
\begin{aligned}
& \left|\frac{\psi(a)+\psi(b)}{2}-\frac{\Gamma(\mu+1)}{2(\phi(b)-\phi(a))^{\mu}}\left(J_{\phi(a)^{+}}^{\mu} \psi(b)+J_{\phi(b)^{-}}^{\mu} \psi(a)\right)\right| \\
& \quad \leq \frac{|\phi(b)-\phi(a)|}{2^{\frac{1}{q}}(\mu+1)}\left(1-\frac{1}{2^{\mu}}\right)\left(\left|\left(\psi \circ \phi^{-1}\right)^{\prime}(\phi(a))\right|^{q}+\left|\left(\psi \circ \phi^{-1}\right)^{\prime}(\phi(b))\right|^{q}\right)^{\frac{1}{q}} .
\end{aligned}
$$

Proof We divide the proof into two cases.

Case 1: $q=1$. By using the property of the absolute value function and convexity of $\left|\left(\psi \circ \phi^{-1}\right)^{\prime}\right|$ in Lemma 1, inequality (2.4) is obtained.

Case 2: $q>1$. By using the property of the absolute value function and power mean inequality on the right-hand side of identity (2.1), the following inequality is established:

$$
\begin{aligned}
& \left|\frac{\psi(a)+\psi(b)}{2}-\frac{\Gamma(\mu+1)}{2(\phi(b)-\phi(a))^{\mu}}\left(J_{\phi(a)^{+}}^{\mu} \psi(b)+J_{\phi(b)^{-}}^{\mu} \psi(a)\right)\right| \\
& \leq \frac{|\phi(b)-\phi(a)|}{2}\left(\int_{0}^{1}\left|(1-t)^{\mu}-t^{\mu}\right|\right)^{1-\frac{1}{q}} \\
& \quad \times\left(\int_{0}^{1}\left|(1-t)^{\mu}-t^{\mu}\right|\left|\left(\psi \circ \phi^{-1}\right)^{\prime}(t \phi(a)+(1-t) \phi(b))\right|^{q} d t\right)^{\frac{1}{q}} .
\end{aligned}
$$

We have that

$$
\begin{aligned}
\int_{0}^{1}\left|(1-t)^{\mu}-t^{\mu}\right| d t & =\int_{0}^{\frac{1}{2}}\left((1-t)^{\mu}-t^{\mu}\right) d t+\int_{\frac{1}{2}}^{1}\left(t^{\mu}-(1-t)^{\mu}\right) d t \\
& =\frac{2}{(\mu+1)}\left(1-\frac{1}{2^{\mu}}\right) .
\end{aligned}
$$

Also, by convexity of $\left|\left(\psi \circ \phi^{-1}\right)^{\prime}\right|^{q}$, we get the following inequality:

$$
\begin{aligned}
& \int_{0}^{1}\left|(1-t)^{\mu}-t^{\mu}\right|\left|\left(\psi \circ \phi^{-1}\right)^{\prime}(t \phi(a)+(1-t) \phi(b))\right|^{q} d t \\
& \leq \int_{0}^{\frac{1}{2}}\left((1-t)^{\mu}-t^{\mu}\right)\left(t\left|\left(\psi \circ \phi^{-1}\right)^{\prime}(\phi(a))\right|^{q}+(1-t)\left|\left(\psi \circ \phi^{-1}\right)^{\prime}(\phi(b))\right|^{q}\right) d t \\
& \quad+\int_{\frac{1}{2}}^{1}\left(t^{\mu}-(1-t)^{\mu}\right)\left(t\left|\left(\psi \circ \phi^{-1}\right)^{\prime}(\phi(a))\right|^{q}+(1-t)\left|\left(\psi \circ \phi^{-1}\right)^{\prime}(\phi(b))\right|^{q}\right) d t \\
& =\left|\left(\psi \circ \phi^{-1}\right)^{\prime}(\phi(a))\right|^{q}\left(\int_{0}^{\frac{1}{2}} t\left((1-t)^{\mu}-t^{\mu}\right) d t+\int_{0}^{\frac{1}{2}} t\left(t^{\mu}-(1-t)^{\mu}\right) d t\right) \\
& \quad+\left|\left(\psi \circ \phi^{-1}\right)^{\prime}(\phi(b))\right|^{q} \\
& \quad \times\left(\int_{\frac{1}{2}}^{1}(1-t)\left((1-t)^{\mu}-t^{\mu}\right) d t+\int_{\frac{1}{2}}^{1}(1-t)\left(t^{\mu}-(1-t)^{\mu}\right) d t\right) .
\end{aligned}
$$


We now compute the integrals appearing on the right-hand side of the above inequality:

$$
\begin{aligned}
& \int_{0}^{\frac{1}{2}} t\left((1-t)^{\mu}-t^{\mu}\right) d t+\int_{0}^{\frac{1}{2}} t\left(t^{\mu}-(1-t)^{\mu}\right) d t=\frac{1}{\mu+1}\left(1-\frac{1}{2^{\mu}}\right) \\
& \int_{\frac{1}{2}}^{1}(1-t)\left((1-t)^{\mu}-t^{\mu}\right) d t+\int_{\frac{1}{2}}^{1}(1-t)\left(t^{\mu}-(1-t)^{\mu}\right) d t=\frac{1}{\mu+1}\left(1-\frac{1}{2^{\mu}}\right)
\end{aligned}
$$

Using (2.9) and (2.10) in (2.8), the required inequality can be obtained.

Corollary 7 By setting $\phi(x)=x$, inequality (2.6) reduces to the following inequality:

$$
\begin{gathered}
\left|\frac{\psi(a)+\psi(b)}{2}-\frac{\Gamma(\mu+1)}{2(b-a)^{\mu}}\left(J_{(a)^{+}}^{\mu} \psi(b)+J_{(b)^{-}}^{\mu} \psi(a)\right)\right| \\
\leq \frac{b-a}{2^{\frac{1}{q}}(\mu+1)}\left(1-\frac{1}{2^{\mu}}\right)\left(\left|\psi^{\prime}(a)\right|^{q}+\left|\psi^{\prime}(b)\right|^{q}\right)^{\frac{1}{q}} .
\end{gathered}
$$

Corollary 8 By setting $\phi(x)=\frac{1}{x}$, inequality (2.6) reduces to the following inequality:

$$
\begin{aligned}
& \left|\frac{\psi(a)+\psi(b)}{2}-\frac{\Gamma(\mu+1)}{2}\left(\frac{a b}{b-a}\right)^{\mu}\left(J_{\left(\frac{1}{a}\right)^{-}}^{\mu} \psi \circ g\left(\frac{1}{b}\right)+J_{\left(\frac{1}{b}\right)^{+}}^{\mu} \psi \circ g\left(\frac{1}{a}\right)\right)\right| \\
& \leq \frac{b-a}{2^{\frac{1}{q}} a b(\mu+1)}\left(1-\frac{1}{2^{\mu}}\right)\left(a^{2 q}\left|\psi^{\prime} \phi(a)\right|^{q}+b^{2 q}\left|\psi^{\prime}(b)\right|^{q}\right)^{\frac{1}{q}}
\end{aligned}
$$

Corollary 9 By setting $\phi(x)=\ln x$, inequality (2.6) reduces to the following inequality:

$$
\begin{gathered}
\left|\frac{\psi(a)+\psi(b)}{2}-\frac{\Gamma(\mu+1)}{2(\ln b-\ln a)^{\mu}}\left(J_{(\ln a)^{+}}^{\mu} \psi(u)+J_{(\ln b)^{-}}^{\mu} \psi(a)\right)\right| \\
\leq \frac{\ln b-\ln a}{2^{\frac{1}{q}}(\mu+1)}\left(1-\frac{1}{2^{\mu}}\right)\left(\left(a^{q}\left|\psi^{\prime} \phi(a)\right|^{q}+b^{q}\left|\psi^{\prime}(b)\right|^{q}\right)^{\frac{1}{q}} .\right.
\end{gathered}
$$

Corollary 10 By setting $\phi(x)=x^{r}, r \neq 0$, inequality (2.6) reduces to the following inequality:

$$
\begin{aligned}
& \left|\frac{\psi(a)+\psi(b)}{2}-\frac{r^{\mu} \Gamma(\mu+1)}{2\left(b^{r}-a^{r}\right)^{\mu}}\left({ }^{r} J_{(a)^{+}}^{\mu} \psi(u)+^{r} J_{(b)^{-}}^{\mu} \psi(a)\right)\right| \\
& \quad \leq \frac{\left|b^{r}-a^{r}\right|}{2^{\frac{1}{q}}(\mu+1)}\left(1-\frac{1}{2^{\mu}}\right)\left(a^{(1-r) q}\left|\psi^{\prime} \phi(a)\right|^{q}+b^{(1-r) q}\left|\psi^{\prime}(b)\right|^{q}\right)^{\frac{1}{q}} .
\end{aligned}
$$

The following lemma is useful to prove the next theorem.

Lemma 2 ([19]) For $0<\alpha<1$ and $0 \leq a<b$, we have

$$
\left|a^{\alpha}-b^{\alpha}\right| \leq(b-a)^{\alpha}
$$

Theorem 5 Let $a, b \in \mathbb{R}$ with $a<b$ and $\psi:[a, b] \rightarrow \mathbb{R}$ be a function, also let $\phi:[a, b] \rightarrow \mathbb{R}$ be a strictly monotone function such that $\psi \circ \phi^{-1}$ is differentiable and $\left(\psi \circ \phi^{-1}\right)^{\prime} \in L[a, b]$. 
If $\left|\left(\psi \circ \phi^{-1}\right)^{\prime}\right|^{q}, q>1$ is convex, then the following inequality holds:

$$
\begin{aligned}
& \left|\frac{\psi(a)+\psi(b)}{2}-\frac{\Gamma(\mu+1)}{2(\phi(b)-\phi(a))^{\mu}}\left(J_{\phi(a)^{+}}^{\mu} \psi(b)+J_{\phi(b)^{-}}^{\mu} \psi(a)\right)\right| \\
& \leq \frac{|\phi(b)-\phi(a)|}{2^{1+\frac{1}{q}}(\mu p+1)^{\frac{1}{p}}}\left(\left|\left(\psi \circ \phi^{-1}\right)^{\prime}(\phi(a))\right|^{q}+\left|\left(\psi \circ \phi^{-1}\right)^{\prime}(\phi(b))\right|^{q}\right)^{\frac{1}{q}}
\end{aligned}
$$

where $\frac{1}{p}+\frac{1}{q}=1$.

Proof By using the property of the absolute value function and then Hölder's inequality on the right-hand side of (2.1), the following inequality is obtained:

$$
\begin{aligned}
& \left|\frac{\psi(a)+\psi(b)}{2}-\frac{\Gamma(\mu+1)}{2(\phi(b)-\phi(a))^{\mu}}\left(J_{\phi(a)^{+}}^{\mu} \psi(b)+J_{\phi(b)^{-}}^{\mu} \psi(a)\right)\right| \\
& \leq \frac{|\phi(b)-\phi(a)|}{2}\left(\int_{0}^{1}\left|(1-t)^{\mu}-t^{\mu}\right|^{p}\right)^{\frac{1}{p}} \\
& \quad \times\left(\int_{0}^{1}\left|\left(\psi \circ \phi^{-1}\right)^{\prime}(t \phi(a)+(1-t) \phi(b))\right|^{q} d t\right)^{\frac{1}{q}} .
\end{aligned}
$$

Using Lemma 2, we have

$$
\begin{aligned}
\int_{0}^{1}\left|(1-t)^{\mu}-t^{\mu}\right|^{p} d t & \leq \int_{0}^{1}|1-2 t|^{\mu p} d t \\
& =\int_{0}^{\frac{1}{2}}(1-2 t)^{\mu p} d t+\int_{\frac{1}{2}}^{1}(2 t-1)^{\mu p} d t=\frac{1}{\mu p+1} .
\end{aligned}
$$

Also, by convexity of $\left|\left(\psi \circ \phi^{-1}\right)^{\prime}\right|^{q}$, we get the following inequality:

$$
\begin{aligned}
& \int_{0}^{1}\left|\left(\psi \circ \phi^{-1}\right)^{\prime}(t \phi(a)+(1-t) \phi(b))\right|^{q} d t \\
& \quad \leq \int_{0}^{1}\left(t\left|\left(\psi \circ \phi^{-1}\right)^{\prime}(\phi(a))\right|^{q}+(1-t)\left|\left(\psi \circ \phi^{-1}\right)^{\prime}(\phi(b))\right|^{q}\right) d t \\
& \quad=\frac{\left|\left(\psi \circ \phi^{-1}\right)^{\prime}(\phi(a))\right|^{q}+\left|\left(\psi \circ \phi^{-1}\right)^{\prime}(\phi(b))\right|^{q}}{2} .
\end{aligned}
$$

Hence, by using above calculations of integrals, one can obtain inequality (2.12).

Corollary 11 By setting $\phi(x)=\frac{1}{x}$, inequality (2.12) reduces to the following inequality:

$$
\begin{aligned}
& \left|\frac{\psi(a)+\psi(b)}{2}-\frac{\Gamma(\mu+1)(a b)^{\mu}}{2(b-a)^{\mu}}\left(J_{\left(\frac{1}{a}\right)^{-}}^{\mu} \psi \circ g\left(\frac{1}{b}\right)+J_{\left(\frac{1}{b}\right)^{+}}^{\mu} \psi \circ g\left(\frac{1}{a}\right)\right)\right| \\
& \leq \frac{b-a}{4(\mu p+1)^{\frac{1}{p}} a b}\left\{a^{2 q}\left|\psi^{\prime}(a)\right|^{q}+b^{2 q}\left|\psi^{\prime}(b)\right|^{q}\right\}^{\frac{1}{q}},
\end{aligned}
$$

where $g(t)=\frac{1}{t}$. 
Corollary 12 By setting $\phi(x)=x^{r}, r \neq 0$, inequality (2.12) reduces to the following inequality:

$$
\begin{aligned}
& \left|\frac{\psi(a)+\psi(b)}{2}-\frac{r^{\mu} \Gamma(\mu+1)}{2\left(b^{r}-a^{r}\right)}\left({ }^{r} J_{a^{+}}^{\mu} \psi(b)+{ }^{r} J_{b^{-}}^{\mu} \psi(a)\right)\right| \\
& \leq \frac{\left|b^{r}-a^{r}\right|}{4|r|(p+1)^{\frac{1}{p}}}\left\{a^{q(1-r)}\left|\psi^{\prime}(a)\right|^{q}+b^{q(1-r)}\left|\psi^{\prime}(b)\right|^{q}\right\}^{\frac{1}{q}} .
\end{aligned}
$$

Corollary 13 By setting $\phi(x)=\ln x$, inequality (2.12) reduces to the following inequality:

$$
\begin{aligned}
& \left|\frac{\psi(a)+\psi(b)}{2}-\frac{\Gamma(\mu+1)}{2(\ln b-\ln a)^{\mu}}\left(J_{(\ln a)^{+}}^{\mu} \psi(b)+J_{(\ln b)^{-}}^{\mu} \psi(a)\right)\right| \\
& \leq \frac{\ln b-\ln a}{2(\mu p+1)^{\frac{1}{p}}}\left\{a^{q}\left|\psi^{\prime}(a)\right|^{q}+b^{q}\left|\psi^{\prime}(b)\right|^{q}\right\}^{\frac{1}{q}} .
\end{aligned}
$$

Remark 4 By setting $\phi(x)=x$ and $\mu=1$, inequality (2.12) reduces to the inequality proved in Theorem 2.3 of [6].

Remark 5 By considering other strictly monotone functions in place of $\phi$ in Theorem 4, the corresponding inequalities can be formulated.

In the following we establish another identity to further study the error estimates of inequality (1.4).

Lemma 3 Under the assumptions of Lemma 1, the following identity holds:

$$
\begin{aligned}
& \frac{2^{\mu-1} \Gamma(\mu+1)}{(\phi(b)-\phi(a))^{\mu}}\left[I_{\left(\frac{\phi(a)+\phi(b)}{2}\right)^{+}}^{\mu} \psi(b)+I_{\left(\frac{\phi(a)+\phi(b)}{2}\right)^{-}}^{\mu} \psi(a)\right] \\
& -\psi\left(\phi^{-1}\left(\frac{\phi(a)+\phi(b)}{2}\right)\right) \\
& =\frac{\phi(b)-\phi(a)}{4}\left[\int_{0}^{1} t^{\mu}\left(\psi \circ \phi^{-1}\right)^{\prime}\left(\frac{\phi(a) t}{2}+\left(\frac{2-t}{2}\right) \phi(b)\right) d t\right. \\
& \left.-\int_{0}^{1} t^{\mu}\left(\psi \circ \phi^{-1}\right)^{\prime}\left(\phi(a)\left(\frac{2-t}{2}\right)+\frac{\phi(b) t}{2}\right) d t\right] .
\end{aligned}
$$

Proof Integrating by parts, we have

$$
\begin{aligned}
\int_{0}^{\frac{1}{2}} t^{\mu}\left(\psi \circ \phi^{-1}\right)^{\prime}\left(\frac{t}{2} \phi(a)+\frac{(2-t)}{2} \phi(b)\right) d t \\
=\left.\frac{t^{\mu}\left(\psi \circ \phi^{-1}\right)\left(\frac{t}{2} \phi(a)+\left(\frac{2-t}{2}\right) \phi(b)\right)}{\frac{\phi(a)-\phi(b)}{2}}\right|_{0} ^{1} \\
\quad-\int_{0}^{1} \mu t^{\mu-1} \frac{\left(\psi \circ \phi^{-1}\right)\left(\frac{t}{2} \phi(a)+\left(\frac{2-t}{2}\right) \phi(b)\right) d t}{\phi(a)-\phi(b)} \\
=-\frac{2 \psi\left(\phi^{-1}\left(\frac{\phi(a)+\phi(b)}{2}\right)\right)}{(\phi(b)-\phi(a))} \\
\quad+\frac{2 \mu}{\phi(b)-\phi(a)} \int_{0}^{1} t^{\mu-1}\left(\psi \circ \phi^{-1}\right)\left(\frac{t \phi(a)}{2}+\left(\frac{2-t}{2}\right) \phi(b)\right) d t
\end{aligned}
$$




$$
=-\frac{2 \psi\left(\phi^{-1}\left(\frac{\phi(a)+\phi(b)}{2}\right)\right)}{(\phi(b)-\phi(a))}+\frac{2^{\mu+1} \Gamma(\mu+1)}{(\phi(b)-\phi(a))^{\mu+1}} I_{\left(\frac{\phi(a)+\phi(b)}{2}\right)^{+}}^{\mu} \psi(b) .
$$

Above we have used a change of variables. Also

$$
\begin{aligned}
\int_{0}^{1} t^{\mu}\left(\psi \circ \phi^{-1}\right)^{\prime}\left(\frac{t}{2} \phi(b)+\frac{(2-t)}{2} \phi(a)\right) d t \\
=\frac{2 \psi\left(\phi^{-1}\left(\frac{\phi(a)+\phi(b)}{2}\right)\right)}{2(\phi(b)-\phi(a))} \\
\quad-\frac{2 \mu}{\phi(b)-\phi(a)} \int_{0}^{1} t^{\mu-1}\left(\psi \circ \phi^{-1}\right)\left(\frac{t \phi(a)}{2}+\left(\frac{2-t}{2}\right) \phi(b)\right) d t \\
=\frac{2 \psi\left(\phi^{-1}\left(\frac{\phi(a)+\phi(b)}{2}\right)\right)}{(\phi(b)-\phi(a))}+\frac{2^{\mu+1} \Gamma(\mu+1)}{(\phi(b)-\phi(a))^{\mu+1}} I_{\left(\frac{\phi(a)+\phi(b)}{2}\right)^{-}}^{\mu} \psi(a) .
\end{aligned}
$$

From equations (2.15) and (2.16), identity (2.14) is established.

Remark 6 By setting $\phi(x)=x$, identity (2.14) reduces to Lemma 3 of [21]. By setting $\phi(x)=$ $x$ and $\mu=1$, identity (2.14) reduces to Corollary 1 of [20]. By setting $\phi(x)=x$, identity (2.14) reduces to Lemma 2.1 of [12]. By considering other strictly monotone functions in place of $\phi$, the corresponding identities can be formulated.

By using Lemma 3, we prove the following error estimate of the Hermite-Hadamard inequality (1.4).

Theorem 6 Under the assumption of Theorem 3, the following fractional integral inequality holds:

$$
\begin{aligned}
& \left|\frac{2^{\mu-1} \Gamma(\mu+1)}{(\phi(b)-\phi(a))^{\mu}}\left[I_{\left(\frac{\phi(a)+\phi(b)}{2}\right)^{+}}^{\mu} \psi(b)+I_{\left(\frac{\phi(a)+\phi(b)}{2}\right)^{-}}^{\mu} \psi(a)\right]-\psi\left(\phi^{-1}\left(\frac{\phi(a)+\phi(b)}{2}\right)\right)\right| \\
& \leq \frac{|\phi(b)-\phi(a)|}{4(\mu+1)}\left\{\left|\left(\psi \circ \phi^{-1}\right)^{\prime}(\phi(a))\right|+\left|\left(\psi \circ \phi^{-1}\right)^{\prime}(\phi(b))\right|\right\} .
\end{aligned}
$$

Proof By using the property of the absolute value function and the convexity of $\left|\left(\psi \circ \phi^{-1}\right)^{\prime}\right|$ in Lemma 3, we get

$$
\begin{aligned}
& \left|\frac{2^{\mu-1} \Gamma(\mu+1)}{(\phi(b)-\phi(a))^{\mu}}\left[I_{\left(\frac{\phi(a)+\phi(b)}{2}\right)^{+}}^{\mu} \psi(b)+I_{\left(\frac{\phi(a)+\phi(b)}{2}\right)^{-}}^{\mu} \psi(a)\right]-\psi\left(\phi^{-1}\left(\frac{\phi(a)+\phi(b)}{2}\right)\right)\right| \\
& \leq \frac{|\phi(b)-\phi(a)|}{4}\left[\int_{0}^{1}\left|t^{\mu}\left(\psi \circ \phi^{-1}\right)^{\prime}\left(\frac{\phi(a) t}{2}+\left(\frac{2-t}{2}\right) \phi(b)\right)\right| d t\right. \\
& \left.\quad+\int_{0}^{1}\left|t^{\mu}\left(\psi \circ \phi^{-1}\right)^{\prime}\left(\phi(a)\left(\frac{2-t}{2}\right)+\frac{\phi(b) t}{2}\right)\right| d t\right] \\
& \leq \frac{|\phi(b)-\phi(a)|}{4}\left(\left(\left|\left(\psi \circ \phi^{-1}\right)^{\prime}(\phi(a))\right|+\left|\left(\psi \circ \phi^{-1}\right)^{\prime}(\phi(b))\right|\right) \int_{0}^{1} t^{\mu} d t\right),
\end{aligned}
$$

from which we get inequality (2.17). 
Corollary 14 By setting $\phi(x)=\frac{1}{x}$, inequality (2.17) reduces to the following inequality:

$$
\begin{aligned}
& \left|\frac{2^{\mu-1} \Gamma(\mu+1)(a b)^{\mu}}{(b-a)^{\mu}}\left[I_{\left(\frac{a+b}{2 a b}\right)^{-}}^{\mu} \psi \circ g\left(\frac{1}{b}\right)+I_{\left(\frac{a+b)}{2 a b}\right)^{+}}^{\mu} \psi \circ g\left(\frac{1}{a}\right)\right]-\psi\left(\frac{2 a b}{a+b}\right)\right| \\
& \quad \leq \frac{(b-a)(\mu+2)}{4(\mu+1)^{2} a b}\left\{a^{2}\left|\psi^{\prime}(a)\right|+b^{2}\left|\psi^{\prime}(b)\right|\right\},
\end{aligned}
$$

where $g(t)=\frac{1}{t}$.

Corollary 15 By setting $\phi(x)=x^{r}, r \neq 0$, inequality (2.17) reduces to the following inequality:

$$
\begin{aligned}
& \left|\frac{2^{\mu-1} \Gamma(\mu+1) r^{\mu}}{\left(b^{r}-a^{r}\right)^{\mu}}\left({ }^{r} I_{\left(\left(\frac{a^{r}+b^{r}}{2}\right)^{\left.\frac{1}{r}\right)^{+}}\right.}^{\mu} \psi(b)+{ }^{r} I_{\left(\left(\frac{a^{r}+b^{r}}{2}\right)^{\left.\frac{1}{r}\right)^{-}}\right.}^{\mu} \psi(a)\right)-\psi\left(\left(\frac{a^{r}+b^{r}}{2}\right)^{\frac{1}{r}}\right)\right| \\
& \quad \leq \frac{\left|b^{r}-a^{r}\right|(\mu+2)}{4(\mu+1)|r|}\left\{a^{(1-r)}\left|\psi^{\prime}(a)\right|+b^{(1-r)}\left|\psi^{\prime}(b)\right|\right\} .
\end{aligned}
$$

Corollary 16 By setting $\phi(x)=\ln x$, inequality (2.17) reduces to the following inequality:

$$
\begin{aligned}
& \left|\frac{2^{\mu-1} \Gamma(\mu+1)}{\ln b-\ln a}\left(I_{\left(\frac{\ln (a)+\ln (b)}{2}\right)^{+}}^{\mu} \psi(b)+I_{\left(\frac{\ln (a)+\ln (b)}{2}\right)^{-}}^{\mu} \psi(a)\right)-\psi\left(\exp \left(\frac{\ln a+\ln b}{2}\right)\right)\right| \\
& \quad \leq \frac{\ln b-\ln a}{4(\mu+1)}\left\{a\left|\psi^{\prime}(a)\right|+b\left|\psi^{\prime}(b)\right|\right\} .
\end{aligned}
$$

Remark 7 By setting $\phi(x)=x$, inequality (2.17) reduces to an inequality of [21]. By setting $\phi(x)=x, \mu=1$, inequality (2.18) reduces to the inequality proved in Theorem 2.2 of [12].

Theorem 7 Under the assumptions of Theorem 4, the following inequality holds:

$$
\begin{aligned}
& \left|\frac{2^{\mu-1} \Gamma(\mu+1)}{(\phi(b)-\phi(a))^{\mu}}\left[I_{\left(\frac{\phi(a)+\phi(b)}{2}\right)^{+}}^{\mu} \psi(b)+I_{\left(\frac{\phi(a)+\phi(b)}{2}\right)^{-}}^{\mu} \psi(a)\right]-\psi\left(\phi^{-1}\left(\frac{\phi(a)+\phi(b)}{2}\right)\right)\right| \\
& \leq \frac{|\phi(b)-\phi(a)|}{2^{2+\frac{1}{q}}(\mu+1)(\mu+2)^{\frac{1}{q}}} \\
& \quad \times\left[\left(\left|\left(\psi \circ \phi^{-1}\right)^{\prime}(\phi(a))\right|^{q}(\mu+1)+\left|\left(\psi \circ \phi^{-1}\right)^{\prime}(\phi(b))\right|^{q}(\mu+3)\right)^{\frac{1}{q}}\right. \\
& \left.\quad+\left|\left(\psi \circ \phi^{-1}\right)^{\prime}(\phi(a))\right|^{q}(\mu+3)+\left(\left|\left(\psi \circ \phi^{-1}\right)^{\prime}(\phi(b))\right|^{q}(\mu+1)\right)^{\frac{1}{q}}\right] .
\end{aligned}
$$

Proof We divide the proof into two cases.

Case 1: $q=1$. By using the property of the absolute value function and the convexity of $\left|\left(\psi \circ \phi^{-1}\right)^{\prime}\right|$ in Lemma 3, inequality (2.17) is obtained. 
Case 2: $q>1$. By using the property of the absolute value function in Lemma 3 and the power mean inequality, the following inequality holds:

$$
\begin{aligned}
& \left|\frac{2^{\mu-1} \Gamma(\mu+1)}{(\phi(b)-\phi(a))^{\mu}}\left[I_{\left(\frac{\phi(a)+\phi(b)}{2}\right)^{+}}^{\mu} \psi(b)+I_{\left(\frac{\phi(a)+\phi(b)}{2}\right)^{-}}^{\mu} \psi(a)\right]-\psi\left(\phi^{-1}\left(\frac{\phi(a)+\phi(b)}{2}\right)\right)\right| \\
& \leq \frac{|\phi(b)-\phi(a)|}{4}\left(\int_{0}^{1} t^{\mu} d t\right)^{1-\frac{1}{q}}\left[\left(\int_{0}^{1}\left|t^{\mu}\left(\psi \circ \phi^{-1}\right)^{\prime}\left(\frac{\phi(a) t}{2}+\left(\frac{2-t}{2}\right) \phi(b)\right)\right|^{q} d t\right.\right. \\
& \left.\left.+\int_{0}^{1}\left|t^{\mu}\left(\psi \circ \phi^{-1}\right)^{\prime}\left(\phi(a)\left(\frac{2-t}{2}\right)+\frac{\phi(b) t}{2}\right)\right|^{q} d t\right)^{\frac{1}{q}}\right] \\
& \leq \frac{|\phi(b)-\phi(a)|}{4(\mu+1)^{1-\frac{1}{q}}} \\
& \times\left[\left(\left|\left(\psi \circ \phi^{-1}\right)^{\prime}(\phi(a))\right|^{q} \int_{0}^{1} \frac{t^{\mu+1}}{2} d t+\left|\left(\psi \circ \phi^{-1}\right)^{\prime}(\phi(b))\right|^{q} \int_{0}^{1}\left(\frac{2-t}{2}\right) t^{\mu} d t\right)^{\frac{1}{q}}\right. \\
& \left.+\left(\left|\left(\psi \circ \phi^{-1}\right)^{\prime}(\phi(a))\right|^{q} \int_{0}^{1}\left(\frac{2-t}{2}\right) t^{\mu} d t+\left|\left(\psi \circ \phi^{-1}\right)^{\prime}(\phi(b))\right|^{q} \int_{0}^{1} \frac{t^{\mu+1}}{2} d t\right)^{\frac{1}{q}}\right] \\
& =\frac{|\phi(b)-\phi(a)|}{2^{2+\frac{1}{q}}(\mu+1)(\mu+2)^{\frac{1}{q}}} \\
& \times\left[\left(\left|\left(\psi \circ \phi^{-1}\right)^{\prime}(\phi(a))\right|^{q}(\mu+1)+\left|\left(\psi \circ \phi^{-1}\right)^{\prime}(\phi(b))\right|^{q}(\mu+3)\right)^{\frac{1}{q}}\right. \\
& \left.+\left|\left(\psi \circ \phi^{-1}\right)^{\prime}(\phi(a))\right|^{q}(\mu+3)+\left(\left|\left(\psi \circ \phi^{-1}\right)^{\prime}(\phi(b))\right|^{q}(\mu+1)\right)^{\frac{1}{q}}\right] \text {. }
\end{aligned}
$$

Hence, inequality (2.18) is proved.

Corollary 17 By setting $\phi(x)=\frac{1}{x}$, inequality (2.18) reduces to the following inequality:

$$
\begin{aligned}
& \left|\frac{2^{\mu-1} \Gamma(\mu+1)(a b)^{\mu}}{(b-a)^{\mu}}\left[I_{\left(\frac{a+b}{2 a b}\right)^{-}}^{\mu} \psi \circ g\left(\frac{1}{b}\right)+I_{\left(\frac{a+b)}{2 a b}\right)^{+}}^{\mu} \psi \circ g\left(\frac{1}{a}\right)\right]-\psi\left(\frac{2 a b}{a+b}\right)\right| \\
& \leq \frac{b-a}{2^{2+\frac{1}{q}}(\mu+1)(\mu+2)^{\frac{1}{q}} a b}\left[\left\{a^{2 q}\left|\psi^{\prime}(a)\right|^{q}(\mu+1)+b^{2 q}\left|\psi^{\prime}(b)\right|^{q}(\mu+3)\right\}^{\frac{1}{q}}\right. \\
& \left.\quad+\left\{a^{2 q}\left|\psi^{\prime}(a)\right|^{q}(\mu+3)+b^{2 q}\left|\psi^{\prime}(b)\right|^{q}(\mu+1)\right\}^{\frac{1}{q}}\right],
\end{aligned}
$$

where $g(t)=\frac{1}{t}$.

Corollary 18 By setting $\phi(x)=x^{r}, r \neq 0$, inequality (2.18) reduces to the following inequality:

$$
\begin{aligned}
& \left|\frac{2^{\mu-1} \Gamma(\mu+1) r^{\mu}}{\left(b^{r}-a^{r}\right)^{\mu}}\left({ }^{r} I_{\left(\left(\frac{a^{r}+b^{r}}{2}\right)^{\frac{1}{r}}\right)^{+}}^{\mu} \psi(b)+{ }^{r} I_{\left(\left(\frac{a^{r}+b^{r}}{2}\right)^{\frac{1}{r}}\right)^{-}}^{\mu} \psi(a)\right)-\psi\left(\left(\frac{a^{r}+b^{r}}{2}\right)^{\frac{1}{r}}\right)\right| \\
& \leq \frac{\left|b^{r}-a^{r}\right|}{2^{2+\frac{1}{q}}(\mu+1)(\mu+2)^{\frac{1}{q}}|r|}\left[\left\{a^{q(1-r)}\left|\psi^{\prime}(a)\right|^{q}(\mu+1)+b^{q(1-r)}\left|\psi^{\prime}(b)\right|^{q}(\mu+3)\right\}^{\frac{1}{q}}\right. \\
& \left.+\left\{a^{q(1-r)}\left|\psi^{\prime}(a)\right|^{q}(\mu+3)+a^{q(1-r)}\left|\psi^{\prime}(b)\right|^{q}(\mu+1)\right\}^{\frac{1}{q}}\right] .
\end{aligned}
$$


Corollary 19 By setting $\phi(x)=\ln x$, inequality (2.18) reduces to the following inequality:

$$
\begin{aligned}
& \left|\frac{2^{\mu-1} \Gamma(\mu+1)}{\ln b-\ln a}\left(I_{\left(\frac{\ln (a)+\ln (b)}{2}\right)^{+}}^{\mu} \psi(b)+I_{\left(\frac{\ln (a)+\ln (b)}{2}\right)^{-}}^{\mu} \psi(a)\right)-\psi\left(\exp \left(\frac{\ln a+\ln b}{2}\right)\right)\right| \\
& \leq \frac{(\ln b-\ln a)}{2^{2+\frac{1}{q}}(\mu+1)(\mu+2)^{\frac{1}{q}}}\left[\left\{a^{q}\left|\psi^{\prime}(a)\right|^{q}(\mu+1)+b^{q}\left|\psi^{\prime}(b)\right|^{q}(\mu+3)\right\}^{\frac{1}{q}}\right. \\
& \left.\quad+\left\{a^{q}\left|\psi^{\prime}(a)\right|^{q}(\mu+3)+b^{q}\left|\psi^{\prime}(b)\right|^{q}(\mu+1)\right\}^{\frac{1}{q}}\right]^{\frac{1}{q}} .
\end{aligned}
$$

Remark 8 By setting $\phi(x)=x$, inequality (2.18) reduces to the inequality proved in Theorem 5 of [21]. By setting $\phi(x)=x, \mu=1=q$, inequality (2.18) reduces to the inequality proved in Theorem 2.2 of [12].

Remark 9 By considering other strictly monotone functions in place of $\phi$ in Theorem 8, the corresponding inequalities can be formulated.

Theorem 8 Under the assumptions of Theorem 5, the following inequality holds:

$$
\begin{aligned}
& \left|\frac{2^{\mu-1} \Gamma(\mu+1)}{(\phi(b)-\phi(a))^{\mu}}\left[I_{\left(\frac{\phi(a)+\phi(b)}{2}\right)^{+}}^{\mu} \psi(b)+I_{\left(\frac{\phi(a)+\phi(b)}{2}\right)^{-}}^{\mu} \psi(a)\right]-\psi\left(\phi^{-1}\left(\frac{\phi(a)+\phi(b)}{2}\right)\right)\right| \\
& \quad \leq \frac{|\phi(b)-\phi(a)|}{4^{1-\frac{1}{p}}(\mu p+1)^{\frac{1}{p}}}\left[\left|\left(\psi \circ \phi^{-1}\right)^{\prime}(\phi(a))\right|+\left|\left(\psi \circ \phi^{-1}\right)^{\prime}(\phi(b))\right|\right] .
\end{aligned}
$$

Proof By using the property of the absolute value function in Lemma 3 and Hölder's inequality, the following inequality is established:

$$
\begin{aligned}
& \left|\frac{2^{\mu-1} \Gamma(\mu+1)}{(\phi(b)-\phi(a))^{\mu}}\left[I_{\left(\frac{\phi(a)+\phi(b)}{2}\right)^{+}}^{\mu} \psi(b)+I_{\left(\frac{\phi(a)+\phi(b)}{2}\right)^{-}}^{\mu} \psi(a)\right]-\psi\left(\phi^{-1}\left(\frac{\phi(a)+\phi(b)}{2}\right)\right)\right| \\
& \quad \leq \frac{|\phi(b)-\phi(a)|}{4}\left(\int_{0}^{1} t^{\mu p} d t\right)^{\frac{1}{p}}\left[\int_{0}^{1}\left|\left(\psi \circ \phi^{-1}\right)^{\prime}\left(\frac{\phi(a) t}{2}+\left(\frac{2-t}{2}\right) \phi(b)\right)\right| d t\right. \\
& \left.\quad+\int_{0}^{1}\left|\left(\psi \circ \phi^{-1}\right)^{\prime}\left(\phi(a)\left(\frac{2-t}{2}\right)+\frac{\phi(b) t}{2}\right)\right| d t\right] .
\end{aligned}
$$

Now, by using the convexity of $\left|\left(\psi \circ \phi^{-1}\right)^{\prime}\right|$ on the right-hand side of the above inequality (2.20), we get the following inequality:

$$
\begin{aligned}
& \left|\frac{2^{\mu-1} \Gamma(\mu+1)}{(\phi(b)-\phi(a))^{\mu}}\left[I_{\left(\frac{\phi(a)+\phi(b)}{2}\right)^{+}}^{\mu} \psi(b)+I_{\left(\frac{\phi(a)+\phi(b)}{2}\right)^{-}}^{\mu} \psi(a)\right]-\psi\left(\phi^{-1}\left(\frac{\phi(a)+\phi(b)}{2}\right)\right)\right| \\
& \leq \frac{|\phi(b)-\phi(a)|}{4}\left(\int_{0}^{1} t^{\mu p} d t\right)^{\frac{1}{p}}\left[\int_{0}^{1}\left|\left(\psi \circ \phi^{-1}\right)^{\prime}\left(\frac{\phi(a) t}{2}+\left(\frac{2-t}{2}\right) \phi(b)\right)\right| d t\right. \\
& \left.\quad+\int_{0}^{1}\left|\left(\psi \circ \phi^{-1}\right)^{\prime}\left(\phi(a)\left(\frac{2-t}{2}\right)+\frac{\phi(b) t}{2}\right)\right| d t\right] \\
& \leq \frac{|\phi(b)-\phi(a)|}{4(\mu p+1)^{\frac{1}{p}}} \\
& \quad \times\left(\left(\left|\left(\psi \circ \phi^{-1}\right)^{\prime}(\phi(a))\right|^{q} \int_{0}^{1} \frac{t}{2} d t+\left|\left(\psi \circ \phi^{-1}\right)^{\prime}(\phi(b))\right|^{q} \int_{0}^{1}\left(\frac{2-t}{2}\right) d t\right)^{\frac{1}{q}}\right.
\end{aligned}
$$




$$
\begin{aligned}
& +\left|\left(\psi \circ \phi^{-1}\right)^{\prime}(\phi(a))\right|^{q} \int_{0}^{1}\left(\frac{2-t}{2}\right) d t+\left(\left(\left|\left(\psi \circ \phi^{-1}\right)^{\prime}(\phi(b))\right|^{q} \int_{0}^{1} t \frac{t}{2} d t\right)^{\frac{1}{q}}\right. \\
\leq & \frac{|\phi(b)-\phi(a)|}{4(\mu p+1)^{\frac{1}{p}}}\left[\left(\frac{\left|\left(\psi \circ \phi^{-1}\right)^{\prime}(\phi(a))\right|^{q}+3\left|\left(\psi \circ \phi^{-1}\right)^{\prime}(\phi(b))\right|^{q}}{4}\right)^{\frac{1}{q}}\right. \\
& \left.\times\left(\frac{3\left|\left(\psi \circ \phi^{-1}\right)^{\prime}(\phi(a))\right|^{q}+\left|\left(\psi \circ \phi^{-1}\right)^{\prime}(\phi(b))\right|^{q}}{4}\right)^{\frac{1}{q}}\right] \\
\leq & \frac{|\phi(b)-\phi(a)|}{4^{1-\frac{1}{p}}(\mu p+1)^{\frac{1}{p}}}\left[\left|\left(\psi \circ \phi^{-1}\right)^{\prime}(\phi(a))\right|+\left|\left(\psi \circ \phi^{-1}\right)^{\prime}(\phi(b))\right|\right] .
\end{aligned}
$$

Here we have used the fact $a^{q}+b^{q} \leq(a+b)^{q}$, for $q>1, a, b \geq 0$. This completes the proof.

Corollary 20 By setting $\phi(x)=\frac{1}{x}$, inequality (2.19) reduces to the following inequality:

$$
\begin{aligned}
& \left|\frac{2^{\mu-1} \Gamma(\mu+1)(a b)^{\mu}}{(b-a)^{\mu}}\left[I_{\left(\frac{a+b}{2 a b}\right)^{-}}^{\mu} \psi \circ g\left(\frac{1}{b}\right)+I_{\left(\frac{a+b)}{2 a b}\right)^{+}}^{\mu} \psi \circ g\left(\frac{1}{a}\right)\right]-\psi\left(\frac{2 a b}{a+b}\right)\right| \\
& \quad \leq \frac{(b-a)}{4^{1-\frac{1}{p}}(\mu p+1)^{\frac{1}{p}} a b}\left[a^{2}\left|\psi^{\prime}(a)\right|+b^{2}\left|\psi^{\prime}(b)\right|\right],
\end{aligned}
$$

where $g(t)=\frac{1}{t}$.

Corollary 21 By setting $\phi(x)=x^{r}$, inequality (2.19) reduces to the following inequality:

$$
\begin{aligned}
& \left|\frac{2^{\mu-1} \Gamma(\mu+1) r^{\mu}}{\left(b^{r}-a^{r}\right)^{\mu}}\left({ }^{r} I_{\left(\left(\frac{a^{r}+b^{r}}{2}\right)^{\left.\frac{1}{r}\right)^{+}}\right.}^{\mu} \psi(b)+{ }^{r} I_{\left(\left(\frac{a^{r}+b^{r}}{2}\right)^{\left.\frac{1}{r}\right)^{-}}\right.}^{\mu} \psi(a)\right)-\psi\left(\left(\frac{a^{r}+b^{r}}{2}\right)^{\frac{1}{r}}\right)\right| \\
& \quad \leq \frac{\left|b^{r}-a^{r}\right|}{4^{1-\frac{1}{p}}(\mu p+1)^{\frac{1}{p}}}\left[a^{1-r}\left|\psi^{\prime}(a)\right|+b^{1-r}\left|\psi^{\prime}(b)\right|\right] .
\end{aligned}
$$

Corollary 22 By setting $\phi(x)=\ln x$, inequality (2.19) reduces to the following inequality:

$$
\begin{aligned}
& \left|\frac{2^{\mu-1} \Gamma(\mu+1)}{(\ln b-\ln a)^{\mu}}\left[I_{\left(\frac{\ln a+\ln b}{2}\right)^{+}}^{\mu} \psi(b)+I_{\left(\frac{\ln a+\ln b)}{2}\right)^{-}}^{\mu} \psi(a)\right]-\psi\left(\exp \left(\frac{\ln a+\ln b}{2}\right)\right)\right| \\
& \quad \leq \frac{\ln b-\ln a}{4^{1-\frac{1}{p}}(\mu p+1)^{\frac{1}{p}}}\left[a\left|\psi^{\prime}(a)\right|+b\left|\psi^{\prime}(b)\right|\right] .
\end{aligned}
$$

Remark 10 By setting $\phi(x)=x$, inequality (2.19) reduces to the inequality proved in Theorem 6 of [21]. By setting $\phi(x)=x, \mu=1$, inequality (2.19) reduces to the inequality proved in Theorem 2.3 of [12].

\section{Conclusions}

The inequalities presented in this paper provide general formulas for fractional versions of trapezoidal and midpoint inequalities for strictly monotone functions. It is noted that the results presented in the published articles $[6,8,10,12,17,20-22]$ can be generated by considering the strictly monotone functions $x, \frac{1}{x}, x^{r}, r \neq 0$, and $\ln x$. The readers can produce corresponding inequalities by taking other strictly monotone functions of their choice. 


\section{Acknowledgements}

This research is partially supported through project KK .01.1.1.02.0027, a project co-financed by the Croatian Government and the European Union through the European Regional Development Fund - the Competitiveness and Cohesion Operational Programme, and by the RUDN University Strategic Academic Leadership Program. And this research is partially supported by the National Science, Research and Innovation Fund (NSRF), Thailand.

Funding

There is no funding available for the publication of this paper.

Availability of data and materials

There is no additional data required for the finding of the results of this paper.

\section{Declarations}

\section{Competing interests}

The authors declare that they have no competing interests.

\section{Authors' contributions}

All authors have equal contribution in this article. All authors read and approved the final manuscript.

\section{Author details}

'Department of Mathematics, COMSATS University Islamabad, Attock Campus, Pakistan. ${ }^{2}$ RUDN University, Miklukho-Maklaya str. 6, 117198 Moscow, Russia. ${ }^{3}$ Department of Mathematics, Faculty of Science, Khon Kaen University, Khon Kaen 40002, Thailand.

\section{Publisher's Note}

Springer Nature remains neutral with regard to jurisdictional claims in published maps and institutional affiliations.

Received: 14 October 2021 Accepted: 28 December 2021 Published online: 24 January 2022

\section{References}

1. Ali, M.A., Alp, N., Budak, H., Chu, Y., Zhang, Z.: On some new quantum midpoint-type inequalities for twice quantum differentiable convex functions. Open Math. 19(1), 427-439 (2021)

2. Budak, H., Khan, S., Ali, M.A., Chu, Y.: Refinements of quantum Hermite-Hadamard-type inequalities. Open Math. 19(1), 724-734 (2021)

3. Cerone, P., Dragomir, S.S.: Trapezoidal type rules from an inequalities point of view. In: Anastassiou, G. (ed.) Handbook of Analytic-Computational Methods in Applied Mathematics, pp. 65-134. CRC Press, New York (2000)

4. Chen, F., Wu, S.: Fejér and Hermite-Hadamard type inequalities for harmonically convex functions. J. Appl. Math. 2014, Article ID 386806 (2014)

5. Dragomir, S.S.: Hermite-Hadamard type inequalities for MN-convex functions. Aust. J. Math. Anal. Appl. 18(1), 1 (2021)

6. Dragomir, S.S., Agarwal, R.P.: Two inequalities for differentiable mappings and applications to special means of real numbers and to trapezoidal formula. Appl. Math. Lett. 11(5), 91-95 (1998)

7. Hadamard, J.: Etude sur les propriétés des fonctions entières et en particulier d'une fonction considérée par Riemann. J. Math. Pures Appl. 58, 171-215 (1893)

8. Iscan, I.: Hermite-Hadamard type inequalities for harmonically convex functions. Hacet. J. Math. Stat. 43(6), 935-942 (2014)

9. Iscan, I.: Hermite-Hadamard type inequalities for $p$-convex functions. Int. J. Anal. Appl. 11(2), 137-145 (2016)

10. Iscan, I., Wu, S.: Hermite-Hadamard type inequalities for harmonically convex functions via fractional integrals. Appl. Math. Comput. 238, 237-244 (2014)

11. Kilbas, A.A., Srivastava, H.M., Trujillo, J.J.: Theory and Applications of Fractional Differential Equations. North-Holland, New York (2006)

12. Kirmaci, U.S.: Inequalities for differentiable mappings and applications to special means of real numbers and to midpoint formula. Appl. Math. Comput. 147, 137-146 (2004)

13. Kunt, M., Iscan, I.: Hermite-Hadamard-Fejér type inequalities for $p$-convex functions. Arab J. Math. Sci. 23, 215-230 (2017)

14. Kunt, M., Iscan, I., Yazici, N., Gozutok, U.: On new inequalities of Hermite-Hadamard-Fejér type for harmonically convex functions via fractional integrals. SpringerPlus 5, 635 (2016)

15. Latif, M.A., Dragomir, S.S., Momoniat, E.: Some Fejér type integral inequalities for geometrically-arithmetically-convex functions with applications. Filomat 32(6), 2193-2206 (2018)

16. Latif, M.A., Hussain, S., Chu, Y.: Generalized Hermite-Hadamard type inequalities for differentiable harmonically-convex and harmonically quasi-convex functions. J. Math. Inequal. 15(2), 755-766 (2021)

17. Noor, M.A., Noor, K.I., Mihai, M.V., Awan, M.U.: Hermite-Hadamard inequalities for differentiable $p$-convex functions using hypergeometric functions (2015) https://www.researchgate.net/publication/282912282. https://doi.org/10.13140/RG.2.1.2485.0648

18. Pečarić, J., Proschan, F., Tong, Y.L.: Convex Functions, Partial Orderings and Statistical Applications. Academic Press, New York (1992)

19. Prudnikov, A.P., Brychkov, I.A., Marichev, O.I.: Integrals and Series: Special Functions, vol. 2. CRC Press, Boca Raton (1986)

20. Sarikaya, M.Z., Set, E., Yaldiz, H., Basak, N.: Hermite-Hadamard's inequalities for fractional integrals and related fractional inequalities. Math. Comput. Model. 57, 2403-2407 (2013) 
21. Sarikaya, M.Z., Yildirim, H.: On Hermite-Hadamard type inequalities for Riemann-Liouville fractional integrals. Miskolc Math. Notes 17(2), 1049-1059 (2017)

22. Toplu, T., Set, E., Iscan, I., Maden, S.: Hermite-Hadamard-type inequalities for $p$-convex functions via Katugampola fractional integrals. Facta Univ., Ser. Math. Inform. 34(1), 149-164 (2019)

23. Tunc, T., Budak, H., Usta, F., Sarikaya, M.Z.: On new generalized fractional integral operators and related fractional inequalities. Konuralp J. Math. 8(2), 268-278 (2020)

24. Turhan, S., Kunt, M., Iscan, I.: Hermite-Hadamard type inequalities for $M_{\varphi} A$-convex functions. Int. J. Math. Model. Comp. 10(1), 57-75 (2020)

25. Zhou, S.S., Farid, G., Jung, C.Y.: Convexity with respect to strictly monotone function and Riemann-Liouville fractional Fejér-Hadamard inequalities. AIMS Math. 6(7), 6975-6985 (2021)

Submit your manuscript to a SpringerOpen ${ }^{\circ}$ journal and benefit from:

- Convenient online submission

$\checkmark$ Rigorous peer review

- Open access: articles freely available online

- High visibility within the field

- Retaining the copyright to your article

Submit your next manuscript at $\downarrow$ springeropen.com 